\title{
Article \\ Classification and Merging Techniques to Reduce Brokerage Using Multi-Objective Optimization
}

\author{
Dhanalakshmi Bettahalli Kengegowda ${ }^{1}(0)$, Srikantaiah Kamidoddi Chowdaiah ${ }^{2}$, Gururaj Harinahalli Lokesh ${ }^{3, *}$ \\ and Francesco Flammini ${ }^{4}$ (D) \\ 1 Department of CSE, BMS Institute of Technology \& Management, Bangalore 560064, India; \\ dhanalakshmi@bmsit.in \\ 2 Department of CSE, SJB Institute of Technology, Bangalore 560060, India; srikantaiahkc@gmail.com \\ 3 Department of Computer Science and Engineering, Vidyavardhaka College of Engineering, \\ Mysuru 570002, India \\ 4 IDSIA USI-SUPSI, Department of Innovative Technologies, University of Applied Sciences and Arts of \\ Southern Switzerland, 6962 Lugano, Switzerland; francesco.flammini@supsi.ch \\ * Correspondence: gururaj1711@vvce.ac.in
}

Citation: Bettahalli Kengegowda, D.; Kamidoddi Chowdaiah, S.; Lokesh,

G.H.; Flammini, F. Classification and Merging Techniques to Reduce

Brokerage Using Multi-Objective

Optimization. Algorithms 2022, 15, 70.

https://doi.org/10.3390/a15020070

Academic Editor:

Massimiliano Caramia

Received: 30 December 2021

Accepted: 8 February 2022

Published: 21 February 2022

Publisher's Note: MDPI stays neutral with regard to jurisdictional claims in published maps and institutional affiliations.

Copyright: (C) 2022 by the authors. Licensee MDPI, Basel, Switzerland. This article is an open access article distributed under the terms and conditions of the Creative Commons Attribution (CC BY) license (https:// creativecommons.org/licenses/by/ $4.0 /)$.

\begin{abstract}
Cloud computing is concerned with effective resource utilization and cost optimization. In the existing system, the cost of resources is much higher. To overcome this problem, a new model called Classification and Merging Techniques for Reducing Brokerage Cost (CMRBC) is designed for effective resource utilization and cost optimization in the cloud. CMRBC has two benefits. Firstly, this is a cost-effective solution to service providers and customers. Secondly, for every job, virtual machine (VM) creations are avoided to reduce brokerage. The allocation, creation or selection of resources of VM is carried out by broker. The main objective is to maximize the resource utilization and minimize brokerage in cloud computing by using Multi-Objective Optimization (MOO). It considered a multi-attribute approach as it has more than two objectives. Likewise, CMRBC implements efficient resource allocation to reduce the usage cost of resources. The outcome of the experiment shows that CMRBC outperforms 60 percent of reduction in brokerage and 10 percent in response time.
\end{abstract}

Keywords: classification; cloud computing; merging; virtual machine; optimization

\section{Introduction}

In the early days, for the archival of stored data from personal computers, one had to have access to the personal computer. Accessing data globally was much more difficult. In the 1980s, the storage of data was conducted using fixed landline data centers, and these data centers were expensive to build. To access resources from a fixed landline server, one had to have access to the grid; this was time-consuming, and in contrast, the cloud is not restricted to storage alone, but it is also used for computing and networking. In computers and computer networking, the network has a complicated section and the collection of computer systems is affected. The cloud in the diagram signifies the fact that details about the system do not affect one's objectives, and therefore, they hide in a cloud. Details about the computer systems delivering the service do not concern the user. Details of how this happens do not matter, and therefore, the system appears in a cloud. To avoid this, users started to purchase or install software and data centers of their own; this proved to be costly. Once a job is done, if resources are not utilized, this means it is underutilized. Therefore, the usage of resources was not scalable and efficient. Additionally, the cloud is not restricted to storage alone, but it is also used for computing and networking. To overcome these problems, the use of resources on rental or lease-based mechanisms lead to the concept of cloud computing. These facilities have access to resources from any part of the world remotely, and the location of stored data is not known to the user. 
Cloud computing is an emerging technology used to store and remotely retrieve data. Hence, users do not have the botheration of investing in infrastructure. This reduces the cost, time, and energy needed, and its concept is 'pay per use'. An example of this is electricity [1].

Public clouds, private clouds, and hybrid clouds are the three types of cloud computing. Cloud computing provides services such as Infrastructure as a Service (Iaas), Platform as a Service (Paas), and Software as a Service (Saas). Iaas contains a pool of hardware resources such as memory, computing power, and storage capacity that are available for rent/lease. Instances of Iaas are presented by virtual machines (VMs) [2].

Computing can be modeled in two ways. The two-tier model directly communicates between the client and infrastructure service provider. Direct communication leads to a reduction in underutilization [3]. The other model is called the three-tier model, which includes one more tier that acts as a mediator role between the client and the service provider [4]. The mediator is called a broker. The cloud broker plays a dual role in the context of cloud computing. When a broker interacts with a provider, it acts as a client and behaves as a provider when interacting with a customer. This leads to effective resource utilization, but the broker benefits more, and the cost automatically escalates. To overcome this problem of cost escalation, a model is designed to reduce brokerage and thereby reduce the number of VM creations for effective resource utilization, as shown in Figure 1. Multiobjective optimization is mainly concerned with multiple attributes and two objective functions. For example, a consumer tends to minimize the cost of car which looks to have maximum comfort while purchasing a car.

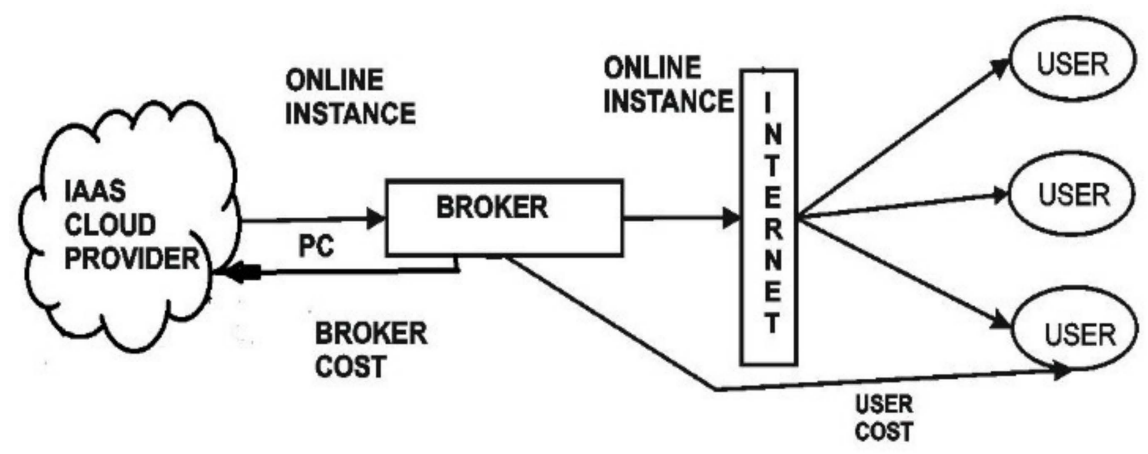

Figure 1. Broker model.

CURA is a Cloud-Managed Model which consists of a VM pool, profiler, and analyzer of a resource management system and works on jobs with deadlines. The VM pool manager, in-turn, assesses the current workload by reconfiguring the cluster workload and accordingly reconfigures the cluster size based on the type of incoming job sizes into small, medium, large, and extra-large and assesses the availability of VM cluster pools. The profiler and analyzer provide the requirements of the incoming job. The profiler identifies the available cluster pool. When smaller jobs arrive and if there is no small cluster pool available, then the available larger cluster pool divides itself into smaller ones. However, in the case of extra-large jobs, if there is no larger cluster pool available, then it cannot fit itself into a smaller cluster pool. This process is known as the reconfiguration of smaller cluster pools. The division and summation of pools is known as the reconfiguration method, which leads to the escalation of cost and is time-consuming. However, to overcome this problem, a CMRBC model is designed. This model refuses to create any type of job pool. Instead of pooling the type of job, it classifies and merges the job together. This avoids brokerage, reduces the number of VM creations and high termination costs, and uses on-demand resources. A summary of the comparison of the existing and proposed model is shown in Table 1. 
Table 1. Summary of the comparison of existing and proposed model.

\begin{tabular}{cccccc}
\hline Authors & Model & Time & RAM & Brokerage & BDW \\
\hline Singh et al. [4] & Per job Optimization & High & High & High & High \\
\hline Anastasiadis et al. [5] & VM-aware Scheduling & Low & High & High & Low \\
\hline Palanisamy et al. [3] & CURA & High & Low & High & Low \\
\hline Proposed Model & CMRBC & Low & Low & Low & High \\
\hline
\end{tabular}

The rest of the paper is organized as follows: related work is described in Section 2, background work is described in Section 3, problem definition is defined in Section 4, the entire structure of the model is described in Section 5, experimental results are described in Section 6, and Section 7 concludes the paper.

\section{Related Works}

Kanu et al. [6] proposed a heuristic scheduling algorithm, Particle Swarm Optimization (PSO), to reduce the total execution cost of application workflow in cloud computing. The Group Arrangement calculation chooses ideal hubs in a dynamic cloud condition to design a bunch to run Map-Reduce programs. The calculation is used for global resource utilization and gives good performance to the customers.

Kessaci et al. [7] explored the request type made by the user and it checked the syntax according to the request type; the scheduler decided which system the request was to be assigned to. Two destinations were considered in the advancement process to limit both the response time and the cost of the VM occurrences, to fulfill the customers to amplify the benefit of the intermediary. The approach was tested by utilizing reasonable information from various Amazon EC2 cases and their pricing histories.

Anastasiadis et al. [5] proposed a new evolutionary algorithm CSA task scheduling in cloud computing. The CSA algorithm is based on optimizing the brokerage and the best-fitting virtual machine for the job. Dynamic procedures are used for the dealer to reserve virtual machines with the target of limiting its service cost. These systems use dynamic programming and guess the calculations to quickly deal with expansive volumes of demands and expand the execution of the resources by reducing response time.

Kumar et al. [8] proposed a DLS algorithm to efficiently trace the workload in cloud computing to meet the requirements of security, time, costs, and executing tasks within the deadline. In this algorithm, a specialist needs to enroll every one of the data centers in a registry. The vitality is devoured by assignment handling, utilizing the virtualization and vitality of IT gear such as switches. The data center chooses a system with the least vitality utilization for assignment handling. This algorithm considers the vitality control relationship and the way that vitality utilization can be reduced.

Valentini et al. [9] proposed some heap load balancing systems depending on both memory and CPU usage, not focused on jobs' execution times. In this system, tasks rely upon a cost grid table which adjusts the heap by sending the job to the allotted virtual machines. Two important requirements that require more consideration for vitality productivity are reducing cost and maximizing resource utilization and system reliability.

Wang et al. [10] proposed dynamic strategies to exploit both pricing advantages of long-term occasion reservations and multiplexing additions to limit cost. A down-to-earth issue confronting cloud clients means limiting their expenses by choosing among various evaluating alternatives because of their requests. Another cloud financier benefit is that it holds an extensive pool of occurrences from cloud suppliers and serves clients with value rebates. The user ideally misuses both estimating advantages of long-term occurrence reservations and multiplexing picks up.

Tiwari et al. [11] designed an algorithm to find the user's needs, the best cloud service provider, and a cloud metaphysics programming algorithm, mainly based on programming techniques. The cloud computing environment provided a convenient broker execution 
management system to bring good prescription measurement programming techniques and a load leveling strategy to allocate resources to a number of customers for their different operations.

Pushpalatha et al. [12] proposed a method for a load balancing scheme to adjust plans for cloud computing to expand the execution of resources by decreasing migration time and response time. The new method with stack-adjusting system TSLB calculation used a compression strategy to increase the usage of resources and to accelerate the procedure. Assignment Portion and Capacity Dispersion are the two issues that should be considered while discussing cloud storage accumulation of VMs.

Marshall et al. [13] proposed a cloud infrastructure that the joins on-request designation of resources with the provision of cycles from sitting out of gear cloud hubs to different procedures by conveying virtual machines (VMs). For this, the Nimbus was utilized. The idea of Optimal Resource Allocation utilizes the thought of HTC clients and on-request clients. A model application that shows the confirmation of the idea was created. The experimental outcomes were encouraging. It decreased the cost of the applications by effectively using cloud resources and found the virtual cloud assets that must be appropriate for every one of the applications.

Aral et al. [14] proposed various novel heuristics, compared them with the round-robin position procedure, and focused on better use of the resources by the cloud environment. In the meantime, Aral et al. minimized the quantity of Application Migration being used, which demonstrated that an application heuristic that depends on the difference between the maximum and minimum usage rates of the resources outperformed another application situation approach and altogether enhanced the conventional methodologies.

He et al. [15] evaluated a new calculation for enhancing the usage of resources for cloud suppliers. The multivariate probabilistic model calculation selected reasonable PMs for VM re-distribution, which were then used to produce a reconfiguration design.

The two heuristics measurements could be utilized as a part of the calculation to enhance the resources' use level for cloud suppliers. An example of this is Amazon EC2, which just gives constrained sorts of VM setups. These groups of VMs are framed by utilizing K-Means bunching calculation. So, before moving to any data centers, sets of VMs are made, and later, they move to the closest data center.

Shakkeera et al. [16] proposed an Optimized Load balancing algorithm in an IaaS virtual cloud environment that limits to use of the virtual cloud resource productively. It limits the cost of the applications by adequately utilizing cloud resources and distinguishes the virtual cloud assets that must be reasonable for every one of the applications. The web application is made with numerous modules. These modules are considered as assignments, and these undertakings are submitted to the load balancing server.

Devi et al. [17] applied a load balancing mechanism to quantify a few QoS execution measurements, i.e., normal execution times, cost, CPU usage, throughput, memory usage, disk space, organize transmission and gathering rate, scheduling success achievement rate, and asset use rate for the number of virtual machines and improve the accessibility among resources using load balancing systems. The essential aim of using assets from the cloud is to minimize the cost and to improve the execution concerning resource usage.

Wang et al. [18] investigated a novel client infrastructure cloud stage, Spot Cloud, through extensive estimations. Supplementing a server, Spot Cloud empowers clients to contribute or pitch their private assets to all in all offer cloud administrations. Even though the limit, as well as the accessibility of this stage, is not yet equivalent to big business data centers, Spot Cloud can give extremely adaptable administrations to clients as far as both execution time and pricing mechanism are concerned. It is neighborly to the clients who frequently look to run here-and-now customer assignments at minimum expenses.

Kumar et al. [19] introduced an innovative idea that provides on-request administration and offers a dynamic registering framework and allots resources in an ideal way. The number of clients requesting assets is expanding nowadays; because of this, it is difficult to designate cloud assets productively and precisely to the clients that fulfill prerequisites and 
the Service Level Agreement (SLA). Numerous parameters can be specified as the factor of the issue that should be considered, such as, for example, stack adjusting, framework throughput, benefit dependability, and benefit-cost.

Mod et al. [20] utilized CloudSim apparatus. CloudSim is an extensible simulation toolbox that empowers the demonstration and recreation of cloud computing frameworks and application provisioning conditions. A few specialists from associations are utilizing CloudSim in their examination of cloud resource provisioning and vitality in the productive administration of server resources. The convenience of CloudSim was affirmed by a contextual analysis, including the dynamic provisioning of utilization benefits for different sorts of resources.

Kumar et al. [21] mentioned the issues with benefits displayed in the current arrangements, including the fact that the cloud supplier may either finish providing resources or may not be in a position to serve a vast number of solicitations. All clients may not require on-request designation. The technique is used with a specific end goal to streamline the resource portion. Numerous applications exist that deal with High-Throughput Figuring (HTF), where more resources are required to be allotted. It permits quick access to servers and the effective usage of accessible assets. This reallocation of VMs enhances the execution of CPU, memory, and system activities by decreasing the heap on data centers.

Aware et al. [22] surveyed cloud computing conditions with different cloud clients to determine the number of cloud benefits in parallel. So, there must be an arrangement in which all assets are made accessible to ask the client for effective ways to fulfill their need. In this overview, an audit of different procedures for dynamic resources allotment in cloud computing is indicated, including the Straight Planning Technique for Asset Assignment, Topology Mindful Asset Portion (TARA), and Dynamic Asset Designation for Parallel Information Handling. Besides restrictions, criticalness and favorable circumstances of utilizing Asset Designation in cloud computing frameworks are likewise discussed.

Panchal et al. [23] concentrate on administrations incorporating IaaS (Framework as an Administration), PaaS (Stage as an Administration), SaaS (Programming as an Administration), and DaaS (Information as an Administration). VM designation enables virtual machines to be shared productively with accessible data centers, and these distribution strategies help to assess and upgrade the cloud execution. Distinctive allotment arrangements are accessible, and they have their points of interest and constraints. A new powerful VM portion arrangement is presented that takes VMs according to client necessity and shares them in a bunch shape to the accessible data centers.

Tripathy et al. [24] used a convention intended to limit the exchanging time, enhance the asset usage, and enhance the server execution and throughput. This technique or convention depends on occupations in the cloud and aims to tackle the downsides in the current conventions. In this convention, the need for the activity which gives better execution to the PC is determined, and attempts are made to limit the holding up time and exchanging time. Efforts have been made to deal with the planning of employment to determine the disadvantages of existing conventions and improve the proficiency and throughput of the server.

Patel et al. [25] proposed a scheduling calculation to beat the proper distribution guide of occupations because of various elements. An orderly survey of different needbased occupation booking calculations was introduced. These calculations are used from an alternate point of view; working standards, etc., infer that all the current procedures essentially center around the need for employment and diminish the benefits to education time and enhancing execution.

Dhanalakshmi et al. [26] developed the extraction of transaction log files to predict multiple output (MOP) in a Multi-Sharing System based on resource utilization for higher accuracy using the prediction techniques Random Forest and majority voting algorithms. The goal was to gratify upcoming resource demands and to avoid over- or under-provisioning of resources. The accuracy results show that the proposed model provides higher accuracy 
in predicting resource utilization for upcoming resource demands, and prediction cost and time are reduced.

In the work of Dhanalakshmi et al. [27], an algorithm named dynamic computation of threshold value (DCTV) was proposed, and based on the threshold value, the jobs were classified in the initial stage, so this classification led to the precise allocation of resources and with efficient resource utilization. The experimental result showed that by using dynamic computation of threshold value, the allocation of resource time was reduced and classification accuracy was improved compared to the manual computation of threshold value.

In the work of Dhanalakshmi et al. [28], the "Multi Sharing Resources in Hybrid Cloud (MSRHC)" model was proposed. The proposed model mainly aimed to enable the sharing of multiple resources by multiple users and provide access control to unused resources. The experimental results showed that by using the Carry Forward of Unused Resources (CFUR) and Access Control for Contributed User (ACCU) approach, the results produced 100 percent of resource utilization and fully fledged access to the contributor user alone, which reduced the cost.

Dhanalakshmi et al. [29] proposed a CMRBC model to minimize brokerage and maximize resource utilization by using classification and merging techniques. This paper is a revised and expanded version of a paper entitled 'Efficient Resource Utilization by Reducing Broker Cost Using Multi-Objective Optimization' presented at the 2nd International Conference on Integrated and Intelligent Computing Communication and Security (IICCS 2018), Bangalore, India, 24-25 January 2018.

\section{Problem Definition}

Given a set ' $J$ ' of ' $n$ ' jobs, where, $J=\left\{j_{1}, j_{2}, j_{3} \ldots j_{n}\right\}$ with different job size, and a set ' $V M$ ' of ' $m$ ' virtual machines, where $V M=\left\{v m_{1}, v m_{2}, v m_{3} \ldots v m_{m}\right\}$, jobs in ' $J$ ' are classified into small, medium, large, and extra-large based on the threshold value $50 \mathrm{MB}, 100 \mathrm{MB}$, $150 \mathrm{MB}, 200 \mathrm{MB}$, and above for the job size, respectively. Once classification is complete, it starts merging smaller jobs based on $\mathrm{VM}$ capacity. The profiler gives a set of requirements ' $P$ ' to execute a job, where $P=\{$ Memory, $C P U, R A M$, Bandwidth, Start time and End time $\}$. Profiler information is submitted to the broker, and in turn, the broker interacts between the user and service provider. The broker assigns the tasks to virtual machines by satisfying all the requirements in ' $P$ '. The broker is the one who is responsible for creating and destroying the virtual machines.

The objective of this work is to maximize resource utilization and minimize the brokerage in cloud computing by using multi-objective optimization.

Assumption: The execution time of every job is assumed to be predicted based on the derived results of profiling, and virtual machine capacity is assumed to be $200 \mathrm{MB}$.

\section{CMRBC: System Model}

The system architecture consists of the following components: users, classifier, merger, mapper, profiler and analyzer, cloudlet, broker, and data center, as shown in Figure 2.

- Users: There are umpteen users who submit their work in the form of jobs to the cloud service provider, and in turn, these jobs are sent to the classifier.

- Classifier: The classifier classifies the jobs into small, medium, large or extra-large based on the threshold value, and classified small jobs are submitted to the merger.

- Merger: The merger segregates the small jobs based on their types. It reads small jobs in each group one by one and starts finding larger_jobs by merging small jobs under consideration if larger_jobs' size is less than or equal to VM capacity. Once the larger_job's size reaches VM capacity, it stops merging, and the larger_job is submitted to the profiler and starts creating a new larger_job for the next small job in the group.

- Profiler and analyzer: The main goal of profiling is to reduce time and error. The profiler and analyzer analyze the job if it arrives for the first time and provides a set of the requirements such as Memory, CPU, RAM, Bandwidth, Start-time, and End-time 
to the mapper for the job to be executed, and these requirements are saved against the job. If the same type of job arrives, its requirements are retrieved and sent to the mapper.

- Mapper: The mapping process makes sure not to violate the SLA constraints, and it is a process of mapping jobs with its requirement to cloudlet by interacting with the local schedulers of each data center after determining the availability of CPU, free time slots, and the expected number of resources to be utilized.

- Cloudlet: The cloudlet is a mobility-enhanced, small-scale cloud data center that is located at the edge of the internet. When a larger_job is submitted to a broker by the mapper, it converts the jobs into tasks with the attributes of job length, type, and time.

- Broker: The broker can act as a negotiator or intermediator between user and service provider; the allocation of a job is performed by the broker to reduce time and effort by assigning the task to available resources in a data center based on users' Quality of Services (QoS). The broker interacts with different CSPs to provide effective resource utilization and decides which task should be executed in which virtual machine. Effective resource assignment strategies and allocation policies are required to satisfy users' needs to maximize the profit for cloud service providers and minimize the cost to cloud users.

- Data Center: In cloud computing, data are not stored on computer hardware nor local machines, but they must be housed on physical drivers somewhere in a data center. A data center is a repository that contains servers, physical machines, virtual machines, and so on, and it can be homogeneous or heterogeneous according to its equipment designs.

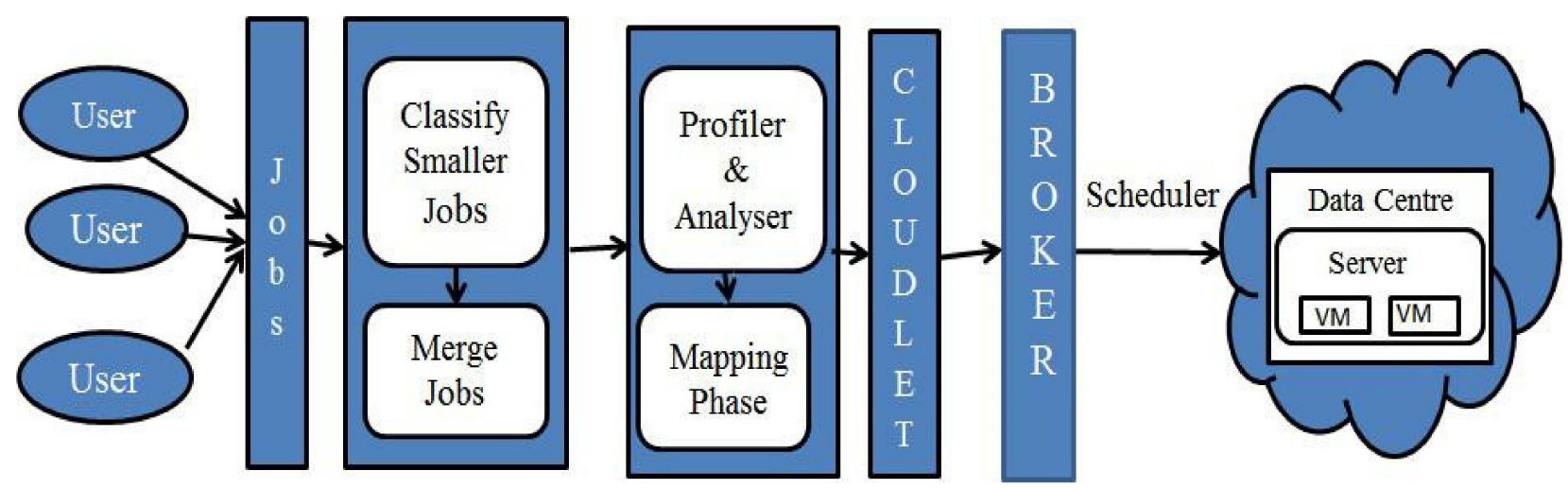

Figure 2. System architecture.

From Table 2, consider a set ' $J$ ' of ' $n$ ' jobs, where $J=\left\{j_{1}, j_{2}, j_{3}, \ldots j_{n}\right\}$, and set ' $V M^{\prime}$ ' of ' $m$ ' virtual machines in the system, where $V M=\left\{v m_{1}, v m_{2}, v m_{3}, \ldots v m_{m}\right\}$. When the request arrives from the cloud user, it is considered as a job, and for job sizes less than or equal to $50,100,150$, and 200, the jobs are classified as small, medium, large, and extra-large, respectively. Since the size of the jobs varies, it is difficult to allocate virtual machines dynamically because the configuration of the virtual machine is the same for all types of jobs and it consumes the same number of resources, for example, the small job does not require a higher-configuration virtual machine. For small jobs, the less resources are required, and for large jobs, more are required. As such, the utilization of resources is not efficient. Hence, to avoid this, the 'classification and merging' method is proposed.

The classification is completed using a classifier. When the classifier receives jobs with different job sizes, it classifies these jobs as small, medium, large, or extra-large jobs based on the threshold value, ' $\zeta$ '. If the job size is less than or equal to $50 \mathrm{MB}$, then it is a small job; if the job size is less than or equal to $100 \mathrm{MB}$, then it is a medium job; if the job size is less than or equal to $150 \mathrm{MB}$, then it is a larger job; if the job size is less than or above $200 \mathrm{MB}$, then it is an extra-large job. 
Table 2. Notations and definitions.

\begin{tabular}{ll}
\hline Notation & Definition \\
\hline$N$ & Total Number of Jobs \\
\hline$M$ & The number of VMs in the system \\
\hline$\zeta$ & Threshold value \\
\hline$C$ & Cost of the larger job \\
\hline$C l$ & The overall cost of the larger job \\
\hline$P j$ & Resources per jobs \\
\hline$\Psi$ & Profiled jobs \\
\hline$B c$ & Size function \\
\hline$R u$ & Broker cost \\
\hline
\end{tabular}

After classification, the set of small jobs are considered as smaller_Jobs which contains ' $n$ ' number of small jobs and it is denoted in Equation (1):

$$
\text { smaller jobs }=\mathrm{ji} \mid \operatorname{sizeof}(\mathrm{ji}) \leq 50
$$

The set of medium jobs lies between 50 to 100 and it is defined as

$$
\text { medium Jobs }=\mathrm{ji} \mid \operatorname{sizeof}\left({ }_{\mathrm{ji}}\right)>50 \text { and } \leq 100
$$

The set of large jobs lies between 100 to 150 and it is defined as

$$
\text { large Jobs } \left.=\mathrm{ji} \mid \operatorname{sizeof}_{\mathrm{ji}}\right)>100 \text { and } \leq 150
$$

The set of extra-large jobs lies between 150 to 200 and it is defined as

$$
\text { extra-large Jobs }=\mathrm{ji} \mid \operatorname{sizeof}(\mathrm{ji}) \leq 200
$$

The set of Smaller_Jobs is given to a merger as input for merging the job, and the merger size is based on the VM capacity.

The jobs in the smaller jobs set are merged until the VM capacity is full, and they become one larger job, and the process repeats. Once the VM capacity is full, it stops merging and repeats the process. The jobs in the set of larger jobs are assigned to one VM.

The order of the Small_jobs is defined as: $\mid$ Smaller_Jobs $\mid=n_{s}$, where $n_{s}$ is an element present in Smaller_Jobs.

The total size of the jobs in the set Smaller_Jobs is given by Equation (5):

$$
\Psi(\text { Smaller_Jobs [1] })+\Psi(\text { Smaller_Jobs [2]) }+\ldots \Psi(\text { Smaller_Jobs }[k]) \leq \Psi(V M)
$$

where $1 \leq K \leq n_{S}$ and ' $\Psi$ ' is a size function.

The jobs in the set Smaller_Jobs are merged until the VM capacity is full, and they become one larger_job, and the process repeats. Once the VM capacity is full, it stops merging and repeats the process. The jobs in the set of larger_jobs are assigned to one VM.

larger_job $[i]=\left\{j_{1} \ldots j_{k} \mid €\right.$ size of $j_{1} \ldots j_{k}$ size $\left.(\Psi)\right\}$

larger_job[I] $=\left\{\left(j_{1} \ldots j_{k}\right) €\right.$ Smaller_Job/Medium_Jobs/Larger_Jobs/Ex-large_Jobs $\left.\mid \operatorname{size}\left(j_{1} \ldots j_{k}\right) \leq \operatorname{size}(V M I)\right\}$

The output of merger is a set of (larger_jobs) and it is given as an input to the profiler; the profiler analyzes the larger_jobs one by one and determines how many resources are required to execute each job in larger_job. The one larger_job contains a set of jobs, i.e., $\left\{j_{1}\right.$, $\left.j_{2} \ldots j_{k}\right\}$; the profiler takes one larger_job and analyzes it one by one and provides the 
requirements to execute each job for the first time arrival. If the same type of job arrives for the second time, the profiling is not completed and it is directly submitted to the broker. Now, the broker decides to create virtual machines for the set of larger_job based on the job size and timestamp. This is shown in Algorithms 1 and 2:

$$
\left(X_{i j}\right)= \begin{cases}0, & \text { if } V M \text { is not created for jobij } \\ 1, & \text { if VM is created for jobij }\end{cases}
$$

where $i=i$ th larger_job, $j=j$ th job in $i$ th larger_job.

Where $x_{i j}$ is equal to 1 , then a virtual machine is created; if $x_{i j}$ equal to 0 , then a VM is not created. The broker cost calculation is the important phase of this model. Brokerage is directly proportional to the number of virtual machines created. The time taken to create and destroy a VM degrades the resource utilization. The cost of each larger job when a VM is created is given by:

$$
\text { Cost }=x_{i j} \times \text { Cost of job }
$$

where $j_{i j}=j$ th $j o b$ in $i$ th larger job. After obtaining the cost of one larger job, the overall cost of resources is calculates, defined as

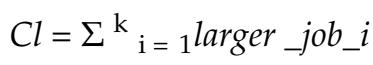

The number of merged larger jobs reduces the number of virtual machines created which impacts the brokerage and reduces the overall cost. Once the cost of larger jobs is derived, then we need to calculate the broker cost and number of resources, utilized as

$$
B_{c}=K \times c
$$

where $B_{c}$ is broker cost, $c$ is a constant brokerage cost of the creation of one $\mathrm{VM}$, and $K$ is the number of virtual machines created.

$$
\text { Overallexecution Cost }=B_{c}+\text { Overallcostofresources }
$$

The number of virtual machine creations are reduced by using our algorithm ( $n$ $-K)$, the brokerage is reduced by $(n-K) * c / 100$, and the overall execution cost is reduced by $(n-K)$. The CMRBC model utilizes $100 \%$ of resource utilization by creating one larger job which contains a set of jobs, but a virtual machine is only created for larger jobs; so, by doing this we can reduce $60 \%$ of brokerage and $60 \%$ of the overall execution cost. With CMRBC, the resource utilization is $100 \%$ higher than without CMRBC. A multi-objective problem is the optimization of resources, and the goal is to optimize two objective functions simultaneously, such as minimizing broker cost and maximizing resource utilization. Optimization is a linear programming technique subject to equality and inequality constrains. Every linear problem corresponds to another linear program problem called its dual. The original problem is called the primal. To solve Equations (9) and (10) with two objective functions simultaneously, the Multi-Objective Optimization technique is applied as

$$
\text { Minimize_overall_execution }(\mathrm{Cl}): \sum_{\mathrm{i}}^{120} \mathrm{c}_{\mathrm{i}} \mathrm{x}_{\mathrm{ij}} \text { Subjectto } \Sigma^{\text {length } \cdot \text { larger jobi }} \mathrm{j}_{\mathrm{ij}} \leq \Psi(\mathrm{V} \mathrm{Mi})
$$

where $i=1$ to $\mathrm{K}$ and $j=1$ to the length of a larger job. Thus, the objective of the proposed system to minimize the brokerage and maximize the resource utilization is achieved. The detailed algorithm for CMRBC is shown in Algorithm 3. Every job in the algorithm is initially classified as a job with smaller job sizes based on threshold value. Then, using these jobs, it merges and profiles the merge jobs and gives the requirement for each job execution. For every service requested by the user, the broker assigns the jobs to the service provider for the execution of the job. Then, the service provider returns the job to the broker. 

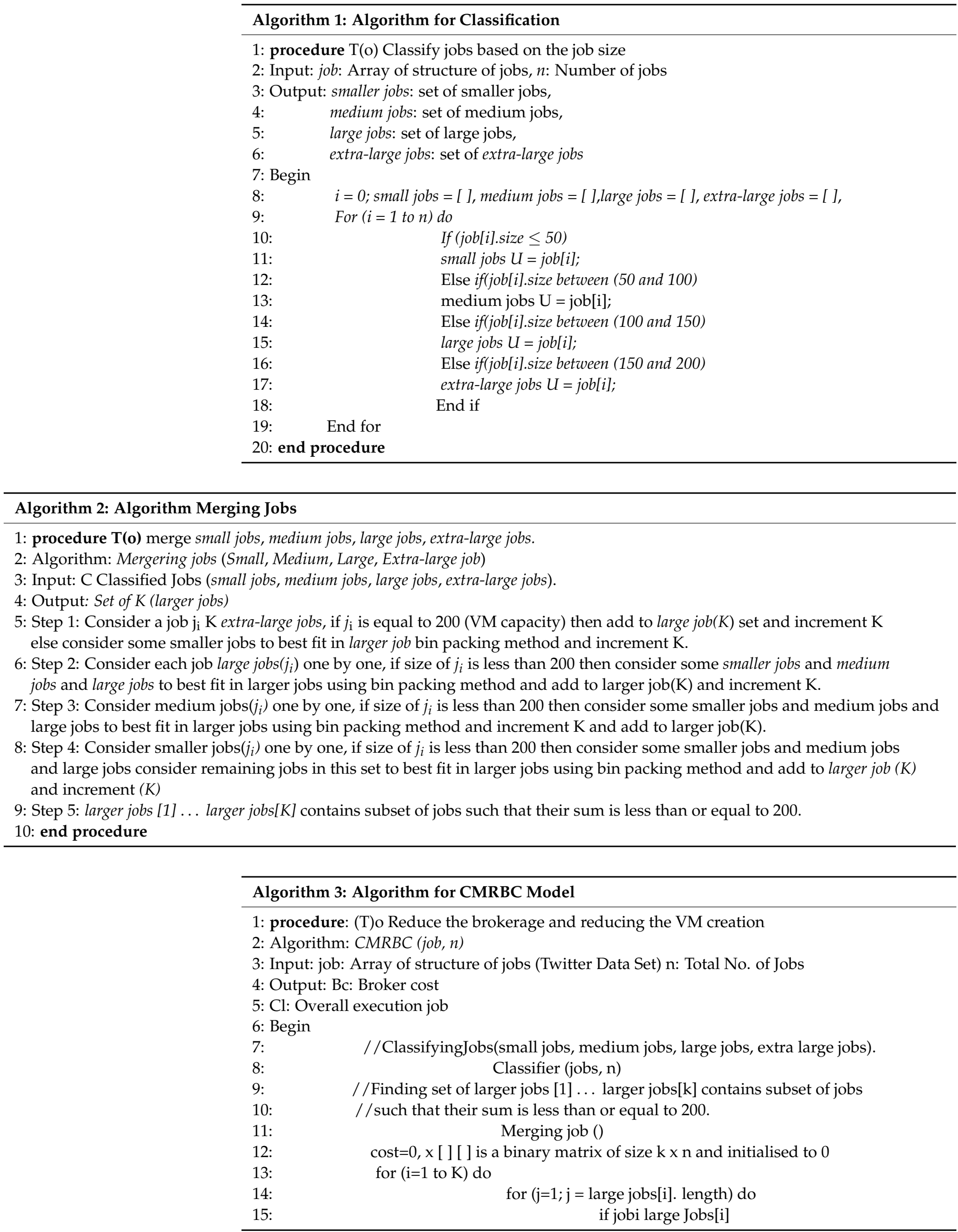
Example: Figure 3 shows the complete structure of the classification and merging process. It considers 10 jobs submitted by the user to a classifier as $\left\{j_{1}, j_{2}, j_{3}, j_{4}, j_{5}, j_{6}, j_{7}, j_{8}, j_{9}\right.$, $\left.j_{10}\right\}$, as shown in Figure 3a. The classifier results in classifying the job as small, medium, large, or extra-large based on threshold value ' $p$ ', where ' $p$ ' is set to $50 \mathrm{MB}$ job size, as shown in Figure $3 b$. Results of the classifier are given as input to the merger, and it first segregates the jobs based on job type, as shown in Figure 3c. Then, it starts merging one by one until it becomes one larger_job, as shown in Figure 3d. Each larger_job is profiled and analyzed to obtain a set of requirements such as CPU Cycles usage, Memory, Bandwidth, Start-time, and End-time and is submitted to a mapper. The mapper interacts with the local scheduler and gives information such as CPU, free time slots, and the expected amount of resources to be utilized. Then, all larger_jobs along with their information are given to the cloudlet, and the cloudlet converts jobs into tasks, as shown in Figure 3e; these tasks are submitted to the broker to be assigned to virtual machines with all the requirements for execution, as shown in Figure 3f.

From Equation (9), we can expand it, as shown below:

$$
\begin{gathered}
\mathrm{B}_{\mathrm{c}}=\mathrm{a}_{1 \times 1}+\mathrm{a}_{2 \times 2}+\mathrm{a}_{3} \times 3+\mathrm{a}_{4} \times 4 \\
=1 \times \mathrm{T}_{1}+0 \times \mathrm{T}_{2}+0 \times \mathrm{T}_{3}+0 \times \mathrm{T}_{4} \ldots \leq 0.50 \\
=0 \times \mathrm{T}_{1}+1 \times \mathrm{T}_{2}+0 \times \mathrm{T}_{3}+0 \times \mathrm{T}_{4} \ldots \leq 0.75 \\
=0 \times \mathrm{T}_{1}+0 \times \mathrm{T}_{2}+1 \times \mathrm{T}_{3}+0 \times \mathrm{T}_{4} \ldots \leq 0.90 \\
\mathrm{~B}_{\mathrm{c}}=0 \times \mathrm{T}_{1}+0 \times \mathrm{T}_{2}+0 \times \mathrm{T}_{3}+1 \times \mathrm{T}_{4} \ldots \leq 0.120
\end{gathered}
$$

The cost of the jobs is considered from Amazon Web Services EC2 (AWS), the total number of jobs are 10, and for each VM, the cost in AWS is 0.50, 0.75, 0.90, and 0.120 (USD) based on the size of the job being small, medium, large, and extra-large, respectively, as shown in Figure 3g. The cost for each VM creation is USD 0.10 in AWS. As based on the classified and merging results shown in Figure 3f, only four VMs are created, so the cost is only calculated for the number of VMs created. This optimizes the brokerage cost for VM creation. The CMRBC model reduced the brokerage cost to $60 \%$ of the actual price for a lesser number of VM creations. The total overall cost is USD 0.335 to execute 10 jobs, USD 0.40 is the cost of the creation of VMs for four larger_jobs instead of USD 0.100 for 10 jobs, and resource utilization is maximized to $80 \%$.

$$
\text { Total Cost }=0.335 \$+0.40 \$=0.375 \$
$$

Time Complexity:

For every job, the CMRBC algorithm is applied and executed by using the classifier and merger function to obtain one larger_job. The time taken for the execution is determined as follows.

Let $n$ be the number of Smaller_Jobs present in the larger_job. Let $t$ be the time taken to compute the brokerage and $v$ the time taken to create and destroy the VM. The time complexity function for computing brokerage and the time taken for the creation and destruction of the VM for $n$ jobs is given by the expression $T(n)=n \times t \times v$. Therefore, the time complexity is of the order $O(n)$. 


\begin{tabular}{|l|l|l|}
\hline Job & Job size & Job type \\
\hline $\mathrm{J}_{1}$ & $30 \mathrm{MB}$ & Small \\
\hline $\mathrm{J}_{2}$ & $50 \mathrm{MB}$ & Small \\
\hline $\mathrm{J}_{3}$ & $100 \mathrm{MB}$ & Medium \\
\hline $\mathrm{J}_{4}$ & $70 \mathrm{MB}$ & Medium \\
\hline $\mathrm{J}_{5}$ & $20 \mathrm{MB}$ & Small \\
\hline $\mathrm{J}_{6}$ & $55 \mathrm{MB}$ & Medium \\
\hline $\mathrm{J}_{7}$ & $45 \mathrm{MB}$ & Small \\
\hline $\mathrm{J}_{8}$ & $5 \mathrm{MB}$ & Small \\
\hline $\mathrm{J}_{9}$ & $150 \mathrm{MB}$ & Large \\
\hline $\mathrm{J}_{10}$ & $50 \mathrm{MB}$ & Small \\
\hline
\end{tabular}

(a)

\begin{tabular}{|c|c|c|}
\hline $\begin{array}{l}\text { Sl } \\
\text { no }\end{array}$ & $\begin{array}{l}\text { Merge } \\
\text { jobs }\end{array}$ & $\begin{array}{l}\text { Job } \\
\text { type }\end{array}$ \\
\hline $\mathrm{M}_{1}$ & $\begin{array}{l}\left\{\mathrm{j}_{1}, \mathrm{j}_{2,}\right. \\
\mathrm{j}_{5}, \quad \mathrm{j} 7 \\
\left.\mathrm{j} 8, \mathrm{j}_{20}\right\}\end{array}$ & $l_{l}$ \\
\hline $\mathrm{M}_{2}$ & $\left\{\mathrm{j}_{4}, \mathrm{j}_{6}\right\}$ & $l_{2}$ \\
\hline $\mathrm{M}_{3}$ & $\left\{j_{3}\right\}$ & $l_{3}$ \\
\hline $\mathrm{M}_{4}$ & $\left\{\mathrm{j}_{g}\right\}$ & $l_{4}$ \\
\hline
\end{tabular}

(d)

\begin{tabular}{|l|l|}
\hline Job no & Job size \\
\hline $\mathrm{J}_{1}$ & $30 \mathrm{MB}$ \\
\hline $\mathrm{J}_{2}$ & $50 \mathrm{MB}$ \\
\hline $\mathrm{J}_{3}$ & $100 \mathrm{MB}$ \\
\hline $\mathrm{J}_{4}$ & $70 \mathrm{MB}$ \\
\hline $\mathrm{J}_{5}$ & $20 \mathrm{MB}$ \\
\hline $\mathrm{J}_{6}$ & $55 \mathrm{MB}$ \\
\hline $\mathrm{J}_{7}$ & $45 \mathrm{MB}$ \\
\hline $\mathrm{J}_{8}$ & $5 \mathrm{MB}$ \\
\hline $\mathrm{J}_{9}$ & $150 \mathrm{MB}$ \\
\hline $\mathrm{J}_{10}$ & $50 \mathrm{MB}$ \\
\hline
\end{tabular}

(b)

\begin{tabular}{|l|l|l|}
\hline $\begin{array}{l}\text { Larger } \\
\text { Job }\end{array}$ & Merge job & Tasks \\
\hline $\mathrm{l}_{1}$ & $\begin{array}{l}\left\{\mathrm{j}_{1}, \mathrm{j}_{5}, \mathrm{j} 7, \mathrm{j} 8,\right. \\
\left.\mathrm{j}_{2}, \mathrm{j}_{10}\right\}\end{array}$ & $\mathrm{T}_{1}$ \\
\hline $\mathrm{l}_{2}$ & $\left\{\mathrm{j}_{4}, \mathrm{j}_{6}\right\}$ & $\mathrm{T}_{2}$ \\
\hline $\mathrm{l}_{3}$ & $\left\{\mathrm{j}_{3}\right\}$ & $\mathrm{T}_{3}$ \\
\hline $\mathrm{l}_{4}$ & $\left\{\mathrm{j}_{9}\right\}$ & $\mathrm{T}_{4}$ \\
\hline
\end{tabular}

(e)

\begin{tabular}{|l|l|l|}
\hline $\begin{array}{l}\text { Sl } \\
\text { no }\end{array}$ & $\begin{array}{l}\text { Segregation } \\
\text { of jobs }\end{array}$ & Job type \\
\hline 1 & $\begin{array}{l}\left\{\mathrm{j}_{l}, \mathrm{j}_{2,} \mathrm{j}_{5}, \mathrm{j}, \mathrm{j}, \mathrm{j},\right. \\
\mathrm{j} 10\}\end{array}$ & Small job \\
\hline 2 & $\left\{\mathrm{j}_{3}, \mathrm{j}_{4}, j_{6}\right\}$ & Medium job \\
\hline 3 & $\{\mathrm{j} 9\}$ & Large job \\
\hline
\end{tabular}

(c)

\begin{tabular}{|l|l|l|}
\hline Tasks & Merge job & $\begin{array}{l}\text { Merged } \\
\text { Job }\end{array}$ \\
\hline $\mathrm{T}_{1}$ & $\begin{array}{l}\left\{\mathrm{j}_{1}, \mathrm{j}_{5}, \mathrm{j}, \mathrm{j}, \mathrm{j},\right. \\
\left.\mathrm{j}_{2}, \mathrm{j}_{20}\right\}\end{array}$ & $\mathrm{VM}_{1}$ \\
\hline $\mathrm{T}_{2}$ & $\left\{\mathrm{j}_{4}, \mathrm{j}_{6\}}\right.$ & $\mathrm{VM}_{2}$ \\
\hline $\mathrm{T}_{3}$ & $\left\{\mathrm{j}_{3}\right\}$ & $\mathrm{VM}_{3}$ \\
\hline $\mathrm{T}_{4}$ & $\left\{\mathrm{j}_{9}\right\}$ & $\mathrm{VM}_{4}$ \\
\hline
\end{tabular}

(f)

\begin{tabular}{|l|l|l|l|l|l|}
\hline $\mathrm{X}_{\mathrm{i}}$ & $\mathrm{VM}_{1}$ & $\mathrm{VM}_{2}$ & $\mathrm{VM}_{3}$ & $\mathrm{VM}_{4}$ & Cost \\
\hline $\mathrm{T} 1(\mathrm{~S})$ & 1 & 0 & 0 & 0 & 0.50 \\
\hline $\mathrm{T} 2(\mathrm{M})$ & 0 & 1 & 0 & 0 & 0.75 \\
\hline $\mathrm{T} 3(\mathrm{~L})$ & 0 & 0 & 1 & 0 & 0.90 \\
\hline $\mathrm{T} 4(\mathrm{XL})$ & 0 & 0 & 0 & 1 & 0.120 \\
\hline
\end{tabular}

$(\mathrm{g})$

Figure 3. (a) Jobs assigned by users, (b) classifier output, (c) segregating jobs, (d) merger output, (e) cloudlet output, (f) task assignment to virtual machines, (g) cost of each type of task.

\section{Model Evaluation Based on the Scenario}

In this subsection, we discuss a model with an example by considering 500 jobs, 500 virtual machines, and 200 data centers in four different scenarios to evaluate the efficacy of the proposed model and compare the utilization of resources with virtual machines. The configuration of a VM involves CPU cycles (HZ), Memory (MB), and Bandwidth (BPS or MBPS) (CPU executes one or more instruction per clock cycle).

Scenario 1: The number of jobs is more than virtual machines.

If the job arrival rate is higher than the virtual machine creation rate, the utilization of resources leads to the scarcity of virtual machines. As a result, the execution of jobs is delayed, and the error rate is increased. For example, for 500 jobs and $200 \mathrm{VMs}$, merging takes place with some type of job to reduce the number of VMs. When there are fewer VMs, the time required to execute each job is more, which eventually leads to starvations. 
Scenario 2: The rate of the arrival of the same type of job is lower than the number of virtual machines.

If the same type of job does not arrive with the expected timestamp, then each job needs to be executed on a single virtual machine. This leads to expensive brokerage, and it is very time consuming to create and destroy virtual machines, meaning charges are high. For example, if there are 100 jobs and $100 \mathrm{VMs}$, if no jobs arrive as small jobs out of 100 jobs, one job is assigned to one virtual machine, which leads to higher costs.

Scenario 3: There are fewer jobs than virtual machines.

If the number of small jobs is less than the number of VMs, then VMs remain idle, waiting for jobs. This leads to the underutilization of resources. Then, merging those small jobs still reduces the creation of VMs. For example, if there are 10 small jobs and there are $50 \mathrm{VM}$, and out of $50 \mathrm{VMs}$, only 10 are utilized, and $40 \mathrm{VMs}$ remain idle.

Scenario 4: When we merge the number of jobs into one job, if any job in the merged jobs fails to be executed due to some reason, the remaining merged jobs cannot be executed. This leads to the incomplete execution of jobs and also affects the other jobs. For example, $J_{1}$ and $J_{5}$ are merged, and if $J_{1}$ is not executed due to some problem, then $J_{5}$ cannot be executed, even though it is possible to execute it.

\section{Experimental Results}

The experiment was run on a cloud simulator tool, version 3.03, and the configuration of the computer was as follows: CPU (64-bit, Intel Pentium(R) i7 CPU 2.9 GHz) with 16 GB RAM and 2 TB hard disk and Net beans 8.1 editors, coded in java language and Intel Core i7 processor environment, with a 100 GB Memory. The CMRBC algorithm implemented both the scheduling of the instant VM allocation and the classification and merging techniques using a Twitter data set, the size of which was 10 GB, with four jobs types: small, medium, large, and extra-large. The size of the job was predefined as small being $50 \mathrm{MB}$, medium being MB, large being $150 \mathrm{MB}$, and a size greater than 150 was considered an extra-large job. The arrival of a job was based on the Poisson distribution, as shown in Table 3.

Table 3. Example of the classification and merging technique workload of 12 jobs.

\begin{tabular}{ccccccc}
\hline 12 Jobs & Job Size & Classification & $\begin{array}{c}\text { VM } \\
\text { Number }\end{array}$ & $\begin{array}{c}\text { Arrival } \\
\text { Time }\end{array}$ & Deadline & $\begin{array}{c}\text { Execution } \\
\text { Time }\end{array}$ \\
\hline 1 & 10 & Small & VM1 & 0 & 150 & 110 \\
\hline 2 & 50 & Small & VM1 & 0 & 150 & 110 \\
\hline 3 & 200 & Extra-large & VM4 & 0 & 250 & 249 \\
\hline 4 & 100 & Medium & VM3 & 120 & 260 & 252 \\
\hline 5 & 35 & Small & VM1 & 122 & 160 & 158 \\
\hline 6 & 40 & Small & VM2 & 138 & 172 & 172 \\
\hline 7 & 150 & Large & VM5 & 140 & 150 & 149 \\
\hline 8 & 75 & Medium & VM3 & 160 & 175 & 175 \\
\hline 9 & 15 & Small & VM1 & 170 & 152 & 110 \\
\hline 10 & 50 & Small & VM1 & 177 & 280 & 110 \\
\hline 11 & 50 & Small & VM1 & 182 & 190 & 110 \\
\hline 12 & 25 & Small & VM2 & 190 & 200 & 199 \\
\hline
\end{tabular}

The experimental results of a workload for 12 jobs using classification and merging techniques are shown in Table 3, and the internal structure of job allocation to virtual machines is shown in Figure 4. The arrival time and deadline of jobs was given previously, as shown in Table 3. The goal of this model was to classify jobs based on the size of them 
using threshold values and merging the jobs based on the virtual machine capacity. The table shows which classified and merged jobs fit which VM type. The arrival time and deadline time were given as soon as the job is submitted to the classifier. The classification and merging of jobs were only completed for smaller jobs. Jobs 1, 2, 5, 6, 9, 10, 11 were classified as small because the size of each job was less or equal to $50 \mathrm{MB}, \mathrm{Job}_{3}$ was extralarge, where the job size was $200 \mathrm{MB}$, job4,8 were medium, where is the sizes were less than or equal to $100 \mathrm{MB}$, and job 7 and job3 were large and were broken down into three small jobs and assigned toVM4. The obtained results are shown in Table 3.

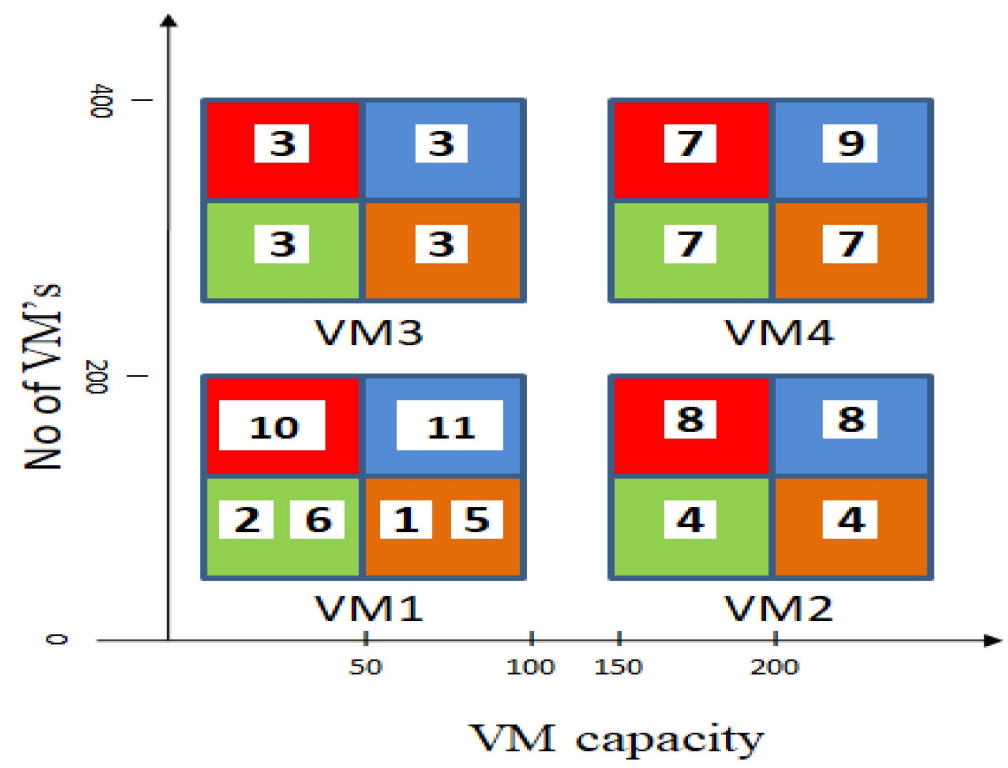

Figure 4. Internal structure of job allocation to virtual machines.

Here, the cost of the broker represents the number of virtual machines that create a job. The CMRBC model enables a reduction in cost for 500 servers because the broker does not necessarily create a VM for every job that is submitted. After all, jobs are already classified and merged to reduce the workload of the broker and to avoid brokerage by creating virtual machines per job.

\section{Performance Evaluation}

The performance of CMRBC is evaluated based on parameters such as the number of servers, resource cost, response time, and resource utilization.

- Several servers: Servers are super-computers that are equipped with programs or hardware of specified configuration which meet certain prerequisites to enable the server to offer services to the computers on its network. Methods that require a relatively lower number of VMs to successfully meet the service quality requirements are not cost-effective. The capital cost is high if a greater number of VMs are used. To overcome this, Table 4 demonstrates the minimum number of servers required to execute the job in the CMRBC model compared to the previously used model, which utilizes a classification and merging technique.

- $\quad$ Response Time: Response time is the total amount of time it takes to respond to a request for service, and it is a sum of service time and waiting time. Systems that have higher response times give poor service quality. Table 4 and Figure 5 demonstrate that the time required to execute a job is less in CMRBC compared to CURA.

- Resource Cost: Resource cost is worked out based on the number of tasks and the number of VMs. In the CURA model, for every 10 tasks, an equal number of VMs are required, and for the CMRBC model, for the same number of tasks, half of the number of VMs are required, i.e., 5 VMs for 10 tasks are required, as shown in Figure 5 and Table 4. 
- Effective Utilization: Here, the effect of distinctive and decided-upon distributions of the deadline for the execution of CMRBC for various values is considered. It was found that both Poisson and uniform distribution require a comparatively fewer number of servers through exponential distribution. This requires 30 percent more servers, as there are more jobs with shorter deadlines in the exponential distribution. Table 4 shows the comparison results of the CURA and CMRBC models with parameters of cost, time, brokerage, RAM speed, and bandwidth.

Table 4. Results of CURA and CMRBC models.

\begin{tabular}{ccccccc}
\hline Model & Cost & Time & Broker RAM & Speed & BDW & Server \\
\hline CURA & 1588 & 946 & 10,564 & 256 & 500 & $1 \mathrm{~GB}$ \\
CMRBC & 1042 & 855 & 8555 & 174 & 300 & $2 \mathrm{~GB}$ \\
\hline
\end{tabular}

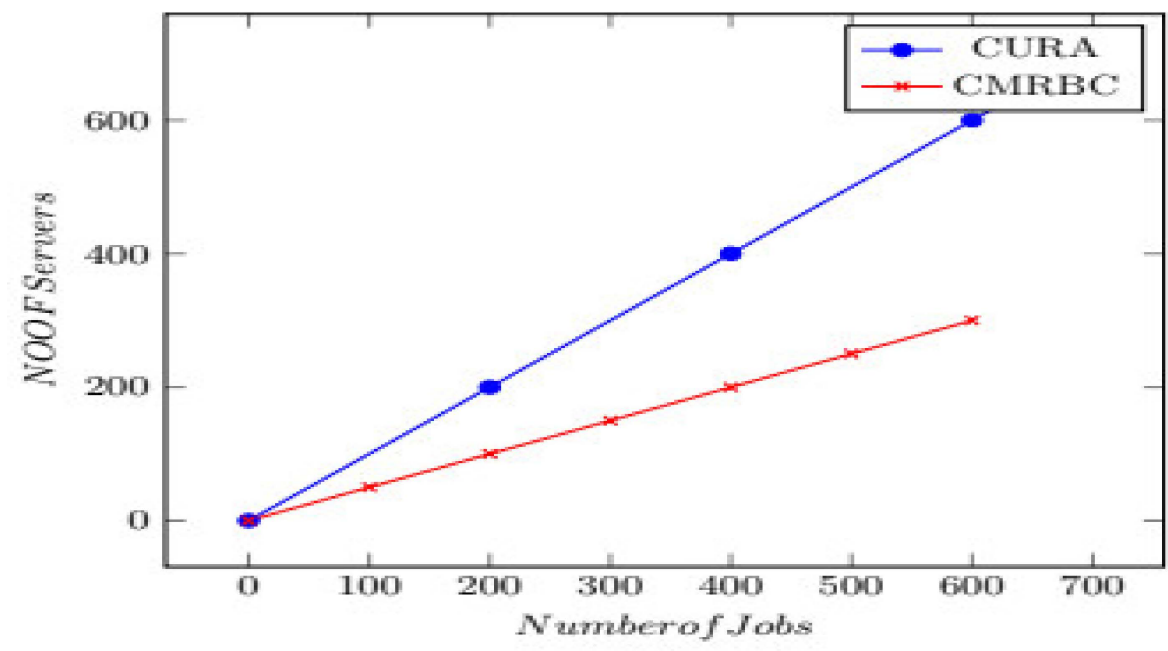

Figure 5. Number of servers required in CURA and CMRBC model.

Considering the cost execution of various classification and merging methodologies, as shown in Figure 4, when requests fluctuate from clients without stable requests, this bends the cost contrast between the online calculations and Heuristic methods. In CMRBC, it is more noticeable when clients have stable requests, but it is difficult to precisely assess their future requests.

1. Number of Jobs Versus Number of servers

The experiments evaluated the performance of the techniques for various job sizes based on the quantum of data input. Small jobs process $50 \mathrm{MB}$ of text data, medium jobs process $100 \mathrm{MB}$, and large and extra-large jobs process 150 and more than $200 \mathrm{~GB}$, respectively. In addition, small, medium, and large jobs have a mean deadline of $50 \mathrm{~s}$, and extra-large jobs have a mean deadline of $180 \mathrm{~s}$, as it takes a bit more running time. It is seen that the performance, in terms of the number of servers required for processing a job under CMRBC, is much lower than the CURA model, as shown in Figure 5.

Improvements were made at a 1:4 ratio for the smaller jobs. A sincere attempt was made to prove that smaller jobs can be executed with minimum time consumption. Hence, it was proved that the CMRBC model requires significantly fewer resources even with peak work-load utilization, as the CURA model demands more resources. The CMRBC model consumes fewer resources, and the output would be at a much more optimal speed, with less consumption of time, and without any hindrance whatsoever. Further, this model will not create virtual machines (thereby reducing their usage) for every small job, because classification and merging techniques are used. 
2. Number of Servers Versus Broker Cost

The comparison between the CURA and CMRBC models is shown in Figure 6. The $x$-axis represents the number of servers, and the $y$-axis represents broker cost.

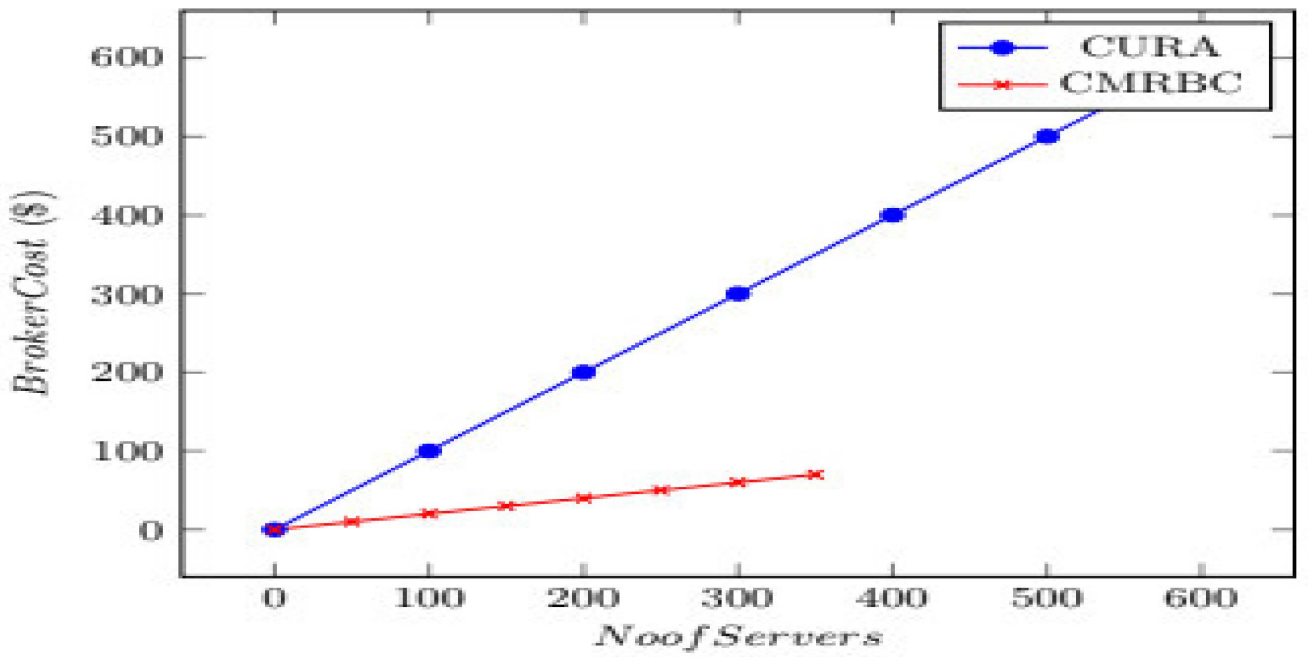

Figure 6. Broker cost comparison between CURA and CMRBC models.

In CURA, to execute each job, one virtual machine is created. The user has to bear the cost for both the creation and termination of the VM, and thus, the cost is on the higher side, and so is the brokerage. In the CMRBC model, for every four jobs, one VM is created, which would automatically reduce the cost of their creation and termination. Hence, the creation ratio is 4, which is towards the cost of VMs. In CURA, four VMs have to be created, ultimately making users pay higher costs. However, in CMRBC, only a one-time cost is levied on the user, thereby reducing the cost to be borne, which amounts to a 4:1 ratio between CURA and CMRBC.

3. Number of Jobs Versus Resource Cost

In Figure 7 , the cost and number of jobs are dealt with. The $x$-axis represents jobs, and the $y$-axis represents resource costs. Here, it is shown how the cost would be reduced by three-fourths. Figure 7 indicates the overall cost involved in executing a bunch of jobs. The CURA model shows a $65 \%$ higher cost than the actual cost incurred by a user under the CMRBC model. This is because of the usage of a lower number of VMs.

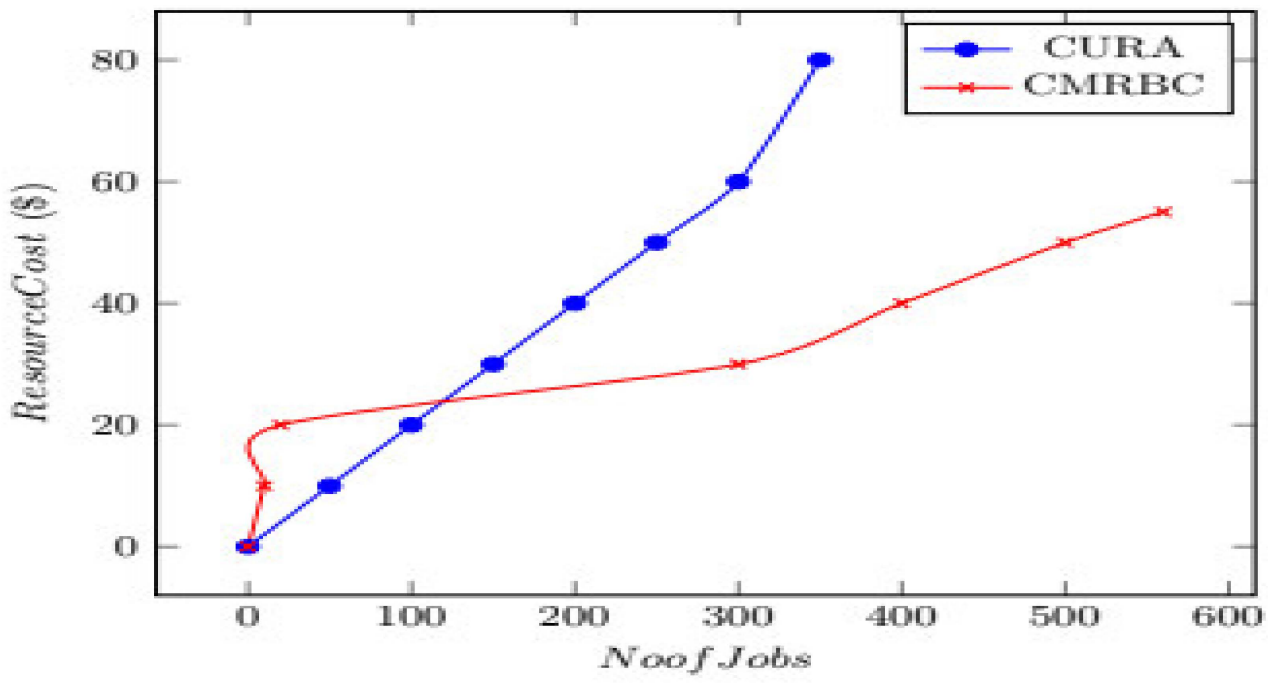

Figure 7. Estimation of cost in CURA and CMRBC models. 
4. Number of Jobs Versus Response Time

In Figure 8, emphasis is laid upon the time consumed for completing jobs, and response time is taken into account. The $x$-axis represents the number of jobs, and the $y$-axis represents response time.

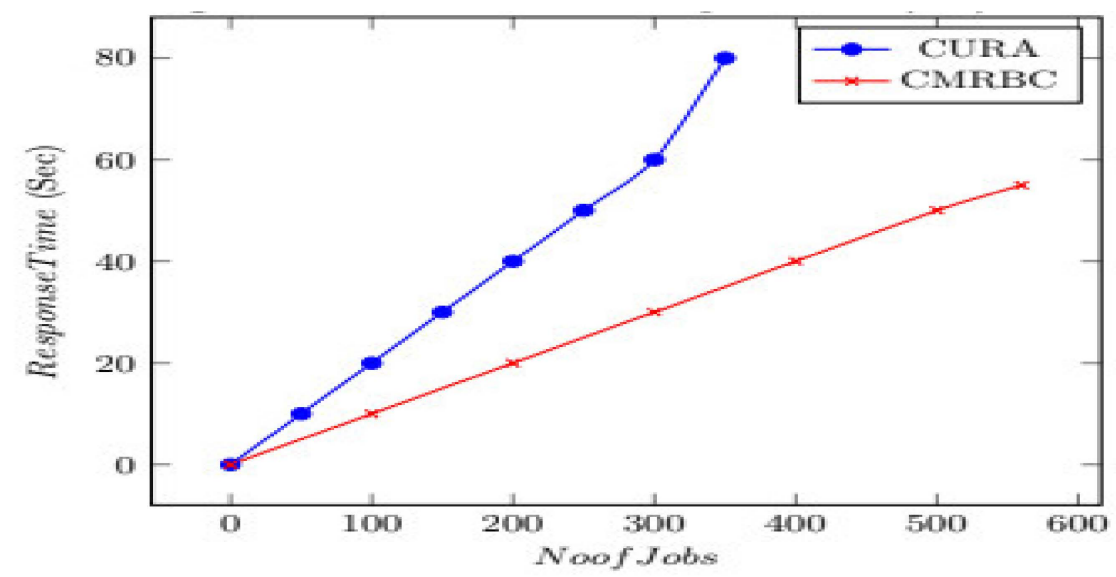

Figure 8. Time required to execute small jobs in CURA and CMRBC models.

A comparison between the CURA model and the CMRBC model is shown. The time consumed for the creation of VMs in the CURA model is double when compared to the CMRBC model. This is due to the creation of fewer VMs. This also automatically reduces the time taken.

\section{Conclusions}

The proposed mechanism, the CMRBC model, was designed to reduce cloud brokerage cost and the overall resource cost using a classification and merging algorithm to optimize the resource cost to satisfy the clients and avoiding either underutilization or overutilization. This provided the efficient utilization of resources to the service provider. It was evaluated using a Poisson distribution in terms of the arrival of jobs for VM requests. The classification setting was completed using threshold values. The experiment was conducted considering the request of 1000 jobs and the request of VMs for four types of instances: small, medium, large, and extra-large. The creation of VMs for each job was avoided. This would automatically reduce brokerage due to the allocation, creation, or selection of VM resources. The CMRBC model was designed to overcome this problem. The results emerged show that the CMRBC model helps to minimize brokerage and maximize effective resource utilization in the above algorithm, to give good results, and to cater to the satisfaction of the clients. The proposed model optimizes two parameters of response, i.e., time and cost, by using multi-objective optimization. By deploying a broker approach, CMRBC does not consider large jobs and extra-large jobs to make resource utilization effective.

Author Contributions: D.B.K. and S.K.C.; methodology, G.H.L. and F.F.; software, G.H.L. and F.F.; validation, D.B.K. and S.K.C.; D.B.K. and S.K.C.; writing-original draft preparation, D.B.K. and S.K.C.; writing-review and editing, G.H.L. and F.F. All authors have read and agreed to the published version of the manuscript.

Funding: This research received no external funding.

Institutional Review Board Statement: Not applicable.

Informed Consent Statement: Not applicable.

Data Availability Statement: Not applicable.

Conflicts of Interest: The authors declare no conflict of interest. 


\section{References}

1. Amazon. Amazon Elastic Compute Cloud API Reference; Amazon: Seattle, WA, USA, 2009.

2. Amazon. Amazon Elastic MapReduce Guide; Amazon: Seattle, WA, USA, 2010.

3. Palanisamy, B.; Singh, A.; Liu, L.; Langston, B. Cura: A Cost-Optimized Model for MapReduce in a Cloud. In Proceedings of the 2013 IEEE 27th International Symposium on Parallel and Distributed Processing, Cambridge, MA, USA, 20-24 May 2013; pp. 1275-1286. [CrossRef]

4. Singh, A.; Korupolu, M.; Mohapatra, D. Server-storage virtualization: Integration and load balancing in data centers. In Proceedings of the 2008 ACM/IEEE Conference on Supercomputing, Austin, TX, USA, 15-21 November 2008 ; pp. 53-67. [CrossRef]

5. Anastasiadis, S.V.; Sevcik, K.C. Parallel application scheduling on networks of workstations. J. Parallel Distrib. Comput. 1997, 43, 109-124. [CrossRef]

6. Kanu, R.P.; Shabeera, T.; Kumar, S.M. Dynamic cluster configuration algorithm in MapReduce cloud. Int. J. Comput. Sci. Inf. Technol. 2014, 5, 4028-4033.

7. Kessaci, Y.; Melab, N.; Talbi, E.-G. A pareto-based genetic algorithm for optimized assignment of VM requests on a cloud brokering environment. In Proceedings of the 2013 IEEE Congress on Evolutionary Computation, Cancun, Mexico, 20-23 June 2013; pp. 2496-2503. [CrossRef]

8. Kumar, B.S.; Parthiban, L. A novel approach for submission of tasks to a data center in a virtualized cloud computing environment. Int. J. Adv. Comput. Sci. Appl. 2016, 7, 238-242.

9. Valentini, G.L.; Lassonde, W.; Khan, S.U.; Min-Allah, N.; Madani, S.A.; Li, J.; Zhang, L.; Wang, L.; Ghani, N.; Kolodziej, J.; et al. An overview of energy efficiency techniques in cluster computing systems. Clust. Comput. 2011, 16, 3-15. [CrossRef]

10. Wang, W.; Niu, D.; Liang, B.; Li, B. Dynamic Cloud Instance Acquisition via IaaS Cloud Brokerage. IEEE Trans. Parallel Distrib. Syst. 2014, 26, 1580-1593. [CrossRef]

11. Tiwari, A.; Sah, M.K.; Gupta, S. Efficient Service Utilization in Cloud Computing Exploitation Victimization as Revised Rough Set Optimization Service Parameters. Procedia Comput. Sci. 2015, 70, 610-617. [CrossRef]

12. Pushpa Latha, K.; Shaji, R.; Jayan, J. A cost-effective load balancing scheme for better resource utilization in cloud computing. J. Emerg. Technol. Web Intell. 2014, 6, 280-290.

13. Marshall, P.; Keahey, K.; Freeman, T. Improving Utilization of Infrastructure Clouds. In Proceedings of the 2011 11th IEEE/ACM International Symposium on Cluster, Cloud and Grid Computing, Newport Beach, CA, USA, 23-26 May 2011; pp. 205-214. [CrossRef]

14. Aral, A.; Ovatman, T. Improving Resource Utilization in Cloud Environments using Application Placement Heuristics. In Proceedings of the International Conference on Cloud Computing and Services Science (CLOSER), Barcelona, Spain, 3-5 April 2014; pp. 527-534. [CrossRef]

15. He, S.; Guo, L.; Ghanem, M.; Guo, Y. Improving resource utilization in the cloud environment using multivariate probabilistic models. In Proceedings of the 2012 IEEE 5th International Conference on Cloud Computing (CLOUD), Honolulu, HI, USA, 24-29 June 2012; pp. 574-581.

16. Shakkeera, L.; Tamilselvan, L.; Imran, M. ImprQoSng resource utilization using QoS based load balancing algorithm or multiple workflows in iaas cloud computing environment. ICTACT J. Commun. Technol. 2013, 4, 750-757.

17. Devi, S.; Gupta, R. An enhancing resource utilization using qos based load balancing algorithm cloud computing. ICTACT J. Commun. Technol. 2014, 4, 21-27.

18. Wang, H.; Wang, F.; Liu, J.; Groen, J. Measurement and utilization of customer-provided resources for cloud computing. In Proceedings of the 2012 Proceedings IEEE INFOCOM, Orlando, FL, USA, 25-30 March 2012; pp. 442-450. [CrossRef]

19. Kumar, D.; Singh, A.S. A survey on resource allocation techniques in cloud computing. In Proceedings of the International Conference on Computing, Communication \& Automation, Greater Noida, India, 15-16 May 2015; pp. 655-660. [CrossRef]

20. Mod, P.; Bhatt, M. Aco based dynamic resource scheduling for improving cloud performance. Int. J. Sci. Eng. Technol. Res. 2014, 3, 3012-3017.

21. Kumar, Y.; Sirisha, K. Optimizing Resource Allocation in IAAS Clouds. Int. J. Computer Trends Technol. 2014, 9, 58-61. [CrossRef]

22. Awasare, V.; Deshmukh, S. Survey and comparative study in resource allocation strategies in the cloud computing environment. IOSR J. Comput. Eng. 2016, 16, 94-101. [CrossRef]

23. Panchal, B.; Kapoor, R. Dynamic VM allocation algorithm using clustering in cloud computing. Int. J. Adv. Res. Comput. Sci. Softw. Eng. 2013, 3, 143-150.

24. Tripathy, L.; Patra, R.R. Scheduling in cloud computing. Int. J. Cloud Comput. Serv. Archit. 2014, 4, 21-27. [CrossRef]

25. Patel, S.; Bhoi, U. Priority based job scheduling techniques in cloud computing: A systematic review. Int. J. Sci. Technol. Res. 2013, 2, 147-152.

26. Dhanalakshmi, B.K.; Srikantaiah, K.C. Predicting Multiple Output in Multi-Sharing System. Int. J. Recent Technol. Eng. (IJRTE) 2019, 8, 4129-4137.

27. Dhanalakshmi, B.K.; Srikantaiah, K.C. Dynamic Computation Threshold value for classifying Jobs in Cloud Computing for efficient Resource Utilization. J. Comput. Theor. Nano Sci. (JCTN) 2020, 17, 4458-4461. [CrossRef] 
28. Dhanalakshmi, B.K.; Srikantaiah, K.C.; Venugopal, K.R. Carry Forward and Access Control for Unused Resources in Multi Sharing System of Hybrid Cloud. Futur. Gener. Comput. Syst. 2020, 110, 282-290. [CrossRef]

29. Dhanalakshmi, B.K.; Srikantaiah, K.C.; Venugopal, K.R. Efficient Resource Utilization by Reducing Broker Cost Using MultiObjective Optimization. In Integrated Intelligent Computing, Communication and Security. Studies in Computational Intelligence; Springer: Singapore, 2019; ISBN 978-981-10-8796-7. 